\title{
Solving a reaction-diffusion system with chemotaxis and non-local terms using Generalized Finite Difference Method. Study of the convergence
}

\author{
J.J. Benito ${ }^{\mathrm{a}, 1}$, A. García ${ }^{\mathrm{a}}$, L. Gavete ${ }^{\mathrm{b}}$, M. Negreanu ${ }^{\mathrm{c}}$, F. Ureña ${ }^{\mathrm{a}, *}$, A.M. \\ $\operatorname{Vargas}^{\mathrm{c}}$ \\ ${ }^{a}$ UNED, ETSII, Madrid, Spain \\ ${ }^{b}$ UPM, ETSIM, Madrid, Spain \\ ${ }^{c}$ Instituto de Matemática Interdisciplinar, Depto. de Análisis Matemático y Matemática \\ Aplicada, UCM, Madrid, Spain
}

\begin{abstract}
In this paper a parabolic-parabolic chemotaxis system of PDEs that describes the evolution of a population with non-local terms is studied. We derive the discretization of the system using the meshless method called Generalized Finite Difference Method. We prove the conditional convergence of the solution obtained from the numerical method to the analytical solution in the twodimensional case. Several examples of the application are given to illustrate the accuracy and efficiency of the numerical method. We also present two examples of a parabolic-elliptic model, as generalized by the parabolic-parabolic system addressed in this paper, to show the validity of the discretization of the non-local terms.
\end{abstract}

Keywords: Chemotaxis system, Generalized Finite Difference, Meshless method, Asymptotic stability.

\section{Introduction}

Chemotaxis is the process under which some living organisms (such as bacterias, cells of the immune system, cells of the endothelium, etc.) direct

\footnotetext{
*Corresponding author

Email addresses: jbenito@ind.uned.es (J.J. Benito), angelochurri@gmail.com (A. García), lu.gavete@upm.es (L. Gavete), negreanu@mat.ucm.es (M. Negreanu), furenaprieto@gmail.com (F. Ureña), antonvar@ucm.es (A.M. Vargas)

Preprint submitted to Journal of Computational and Applied MathematicsDecember 15, 2020
} 
its movement in the direction of a chemical gradient. The individuals of the biological species are able to recognize the chemical signal, to measure its concentration and to move towards the higher concentrations of the substance (positive taxis) or away from it (negative taxis). Mathematical models with chemotactic terms have been applied to model Angiogenesis, a key process in Tumor Growth (see for instance Anderson and Chaplain [1]), in Astrophysics to describe gravitational interaction of particles on the gravitational equilibrium of polytropic stars; in ecology, to describe the attraction of predators to certain chemical signals (pheromons) of the prey in morphogenesis, the creation of shapes and organs in embryonic development, etc.

Since 1970s, chemotaxis has been studied from a mathematical point of view, starting with the first models of PDEs suggested by Keller and Segel [5]. Mathematical bibliography concerning this phenomenon is extensive, although many important results obtained in the area during the last decades can be found in Horstmann [4].

In this paper we consider a parabolic-parabolic system of quasilinear PDEs describing the interactions between the population's density of a biological species, " $U$ ", and the chemical substance, " $V$ ", responsible for the chemotactic process:

$$
\left\{\begin{array}{lr}
\frac{\partial U}{\partial t}=\Delta U-\chi \operatorname{div}\left(U^{m} \nabla V\right)+U\left(a_{0}-a_{1} U^{\alpha}+a_{2} \int_{\Omega} U^{\alpha} d \mathbf{x}\right), & \Omega \times(0, \infty), \\
d \frac{\partial V}{\partial t}=\Delta V-V+U^{\gamma}, & \Omega \times(0, \infty), \\
U(\mathbf{x}, 0)=U_{0}(\mathbf{x}), \quad V(\mathbf{x}, 0)=V_{0}(\mathbf{x}) & \mathbf{x} \in \Omega \\
\frac{\partial U}{\partial \nu}=\frac{\partial V}{\partial \nu}=0 & \partial \Omega \times(0, \infty) .
\end{array}\right.
$$

All the theoretical results of the manuscript are given for the case $d \neq 0$, in particular, without loss of generality, we consider throughout the text $d=1$. For $d=0$, we present in examples 5 and 6 the behavior of the solution, though the proof of the main result of the paper is devoted to the case $d=1$. Model (1) is a generalization of the one considered in [3] where can be found a wide summary of the main results related to (ref1) in the particular case $a_{2}=0$, i.e., the global existence of the solutions and the asymptotic stability of the point $\left(\left(a_{0} / a_{1}\right)^{1 / \alpha},\left(a_{0} / a_{1}\right)^{\gamma / \alpha}\right)$, with $a_{1}$ large enough, are done.

The coefficient " $a_{0}$ ", sometimes also called Malthusian parameter, induces an exponential growth for low density populations if $a_{0}>0$. If $a_{1}>0$, the 
mechanism that limits the growth of biological species $U$ is given by the term $-a_{1} U^{\alpha}$ and it generalizes the most frequent case $\alpha=1$. At the time that the population grows, the competitive effect of the local term $a_{1} U^{\alpha}$ becomes more influential. We also consider a non-local term in the logistic source as $a_{2} \int_{\Omega} U^{\alpha}$ describing the effects of the total mass of the species in the growth of the population. If $a_{2}<0$ there is competition among the individuals of the species for the resources and if $a_{2}>0$, individuals cooperate globally to survive although they compete locally. In the last case, the individuals compete locally but cooperate globally and the effects of $a_{1} U^{\alpha}$ and $a_{2} \int_{\Omega} U^{\alpha}$ balance the system. Non-local terms of such kind have already been used. For instance, in [9] integral terms were used to describe the competition between the cancer cell density and the extracellular matrix density. Also, in [6] authors considered a parabolic-elliptic PDE system with non-local terms and they obtained the global existence of the solutions together with its asymptotic behavior whenever $a_{1}>2 \chi+\left|a_{2}\right|$.

The non-linear nature of the chemotaxis term has been studied in the literature by different authors, see [4] and references therein. The exponent $m$ indicates nonlinearities with respect to $U$ in the tactic sensitivity functions; intuitively, there is a reinforcement of movement in direction of $\nabla V$ where the population $U$ is greater than a normalized value and presents a weaker movement where it is less than it. These terms with $\alpha \geq 1$ induce a negative feedback that slows growth as populations approach their maximum size and a stronger intra-specific concurrence, (via exponents of the involved density).

Due to its importance in applied sciences as Biology, Chemical Engineering and Medicine, and the inherent complexity of the system, because of the non-linearity and non-locality, it is necessary to develop numerical algorithms which are able to solve efficiently system (1).

In this paper we propose the Generalized Finite Difference Method (GFDM). This meshless method has been recently proved to solve with accuracy highly nonlinear PDEs, see [10, 11]. In [2], the authors obtained conditional convergence for the parabolic-elliptic case of system (1) for $d=a_{2}=0, a_{0}=a_{1}$ and $m=\alpha=\gamma=1$. In the cited paper, the conditional convergence of the numerical method is proved in a very different way than in the present paper. Due to the nature of the system, we were able to substitute the terms of the elliptic equation into the parabolic one, which simplified the test. In the present paper we shall use matrix arguments. The differences between the parabolic-elliptic system and the parabolic-parabolic one are also significant 
in the analytical proof of the continuous models, as can be seen in [7] and [8]. Furthermore, the novelty of this paper is the introduction and discretization of non-local terms, which is a challenging task and a problem of growing interest.

The paper is organized as follows: in Section 2 we present some preliminaries of the GFD method, which can be found in $[10,11]$ and the references therein, although for completeness, we mention them below. Section 3 is devoted to the proof of Theorem 3.1, where we find the conditions on the time step of the GFD explicit scheme under which it is convergent. In Section 4 we find the constant steady states of system (1) and present several numerical examples to illustrate its asymptotic stability. In addition, we present some examples of a parabolic-elliptic model to show the validity of the discretization of the non-local terms. We finally present some conclusions.

\section{Preliminaries}

Consider a domain $\Omega \subset \mathbb{R}^{2}$ and let be $M=\left\{\mathbf{x}_{1}, \ldots, \mathbf{x}_{N}\right\} \subset \Omega$ a discretization of it with $N$ points. For each $\mathbf{x}_{0} \in M$, we define $E_{s}=\left\{\mathbf{x}_{0} ; \mathbf{x}_{1}, \ldots, \mathbf{x}_{s}\right\} \subset$ $M$, where $\mathbf{x}_{i}(i=1, \ldots, s)$ can be chosen in several ways, by different criteria. We call $\mathbf{x}_{i}=\left(x_{i}, y_{i}\right)$ and we denote by $h_{i}:=x_{i}-x_{0}$ and $k_{i}:=y_{i}-y_{0}$. Let be $F \in \mathcal{C}^{4}(\Omega)$. Since no confusion with the initial data of $\mathrm{F}$ is possible, we write in this section $F_{0}=F\left(\mathbf{x}_{0}\right)=F\left(x_{0}, y_{0}\right)$ and $F_{i}=F\left(\mathbf{x}_{i}\right)$. By Taylor series expansion, for $i=1, \ldots, s$, we have :

$$
F_{i}=F_{0}+h_{i} \frac{\partial F_{0}}{\partial x}+k_{i} \frac{\partial F_{0}}{\partial y}+\frac{1}{2}\left(h_{i}^{2} \frac{\partial^{2} F_{0}}{\partial x^{2}}+k_{i}^{2} \frac{\partial^{2} F_{0}}{\partial y^{2}}+2 h_{i} k_{i} \frac{\partial^{2} F_{0}}{\partial x \partial y}\right)+\ldots
$$

By ignoring the third and higher order terms in (2), we obtain a second order approximation $f_{i}$ of $F$ in $\mathbf{x}_{i}$. Moreover, we take the vector

$$
\boldsymbol{D}_{5}=\left\{\frac{\partial f_{0}}{\partial x}, \frac{\partial f_{0}}{\partial y}, \frac{\partial^{2} f_{0}}{\partial x^{2}}, \frac{\partial^{2} f_{0}}{\partial y^{2}}, \frac{\partial^{2} f_{0}}{\partial x \partial y}\right\}
$$

In this way, we can obtain an approximation of function $F_{i}$ in terms of the coefficients of $\mathbf{D}_{5}$. In order to determine these, we minimize with respect to the partial derivatives the following function

$$
\begin{aligned}
B(f) & =\sum_{i=1}^{s}\left[\left(f_{0}-f_{i}\right)+h_{i} \frac{\partial f_{0}}{\partial x}+k_{i} \frac{\partial f_{0}}{\partial y}+\right. \\
& \left.+\frac{1}{2}\left(h_{i}^{2} \frac{\partial^{2} f_{0}}{\partial x^{2}}+k_{i}^{2} \frac{\partial^{2} f_{0}}{\partial y^{2}}+2 h_{i} k_{i} \frac{\partial^{2} f_{0}}{\partial x \partial y}\right)\right]^{2} w_{i}^{2}
\end{aligned}
$$


where $w_{i}=w\left(h_{i}, k_{i}\right)$ are positive symmetrical monotone decreasing weighting functions. One arrives then to the following system of linear equations

$$
\boldsymbol{A}\left(h_{i}, k_{i}, w_{i}\right) \boldsymbol{D}_{5}^{T}=\boldsymbol{b}\left(h_{i}, k_{i}, w_{i}, u_{0}, u_{i}\right),
$$

where

$$
\boldsymbol{A}=\left(\begin{array}{cccc}
h_{1} & h_{2} & \cdots & h_{s} \\
k_{1} & k_{2} & \cdots & k_{s} \\
\vdots & \vdots & \vdots & \vdots \\
h_{1} k_{1} & h_{2} k_{2} & \cdots & h_{s} k_{s}
\end{array}\right)\left(\begin{array}{cccc}
\omega_{1}^{2} & & & \\
& \omega_{2}^{2} & \\
& & \cdots & \\
& & & \omega_{s}^{2}
\end{array}\right)\left(\begin{array}{cccc}
h_{1} & k_{1} & \cdots & h_{1} k_{1} \\
h_{2} & k_{2} & \cdots & h_{2} k_{2} \\
\vdots & \vdots & \vdots & \vdots \\
& & & \\
h_{s} & k_{s} & \cdots & h_{s} k_{s}
\end{array}\right)
$$

Some assumptions on the selection criteria of the nodes of $E_{s}$ must be made in order to guarantee that $\mathbf{A}$ is positive definite (see $[10,11]$ for more details on the selection criteria and the weighting functions). By solving system (5), we can find the discretization of the spatial derivatives as functions of $f_{0}$ and $f_{i}$ :

$$
\left\{\begin{array}{l}
\frac{\partial f\left(\mathbf{x}_{0}, n \Delta t\right)}{\partial x}=-m_{01} f_{0}^{n}+\sum_{i=1}^{s} m_{i 1} f_{i}^{n}+\mathcal{O}\left(h_{i}^{2}, k_{i}^{2}\right), \text { with } m_{01}=\sum_{i=1}^{s} m_{i 1} \\
\frac{\partial f\left(\mathbf{x}_{0}, n \Delta t\right)}{\partial y}=-m_{02} f_{0}^{n}+\sum_{i=1}^{s} m_{i 2} f_{i}^{n}+\mathcal{O}\left(h_{i}^{2}, k_{i}^{2}\right), \text { with } m_{02}=\sum_{i=1}^{s} m_{i 2} \\
\frac{\partial^{2} f\left(\mathbf{x}_{0}, n \Delta t\right)}{\partial x^{2}}=-m_{03} f_{0}^{n}+\sum_{i=1}^{s} m_{i 3} f_{i}^{n}+\mathcal{O}\left(h_{i}^{2}, k_{i}^{2}\right), \text { with } m_{03}=\sum_{i=1}^{s} m_{i 3} \\
\frac{\partial^{2} f\left(\mathbf{x}_{0}, n \Delta t\right)}{\partial y^{2}}=-m_{04} f_{0}^{n}+\sum_{i=1}^{s} m_{i 4} f_{i}^{n}+\mathcal{O}\left(h_{i}^{2}, k_{i}^{2}\right), \text { with } m_{04}=\sum_{i=1}^{s} m_{i 4} \\
\frac{\partial^{2} f\left(\mathbf{x}_{0}, n \Delta t\right)}{\partial x \partial y}=-m_{05} f_{0}^{n}+\sum_{i=1}^{s} m_{i 5} f_{i}^{n}+\mathcal{O}\left(h_{i}^{2}, k_{i}^{2}\right), \text { with } m_{05}=\sum_{i=1}^{s} m_{i 5}
\end{array}\right.
$$

Remark 2.1. For simplicity, for the discretization of the laplacian operator, we write

$$
\Delta f\left(\boldsymbol{x}_{0}, n \Delta t\right)=-m_{00} f_{0}^{n}+\sum_{i=1}^{s} m_{i 0} f_{i}^{n},
$$

where $m_{00}=m_{03}+m_{04}$ and $m_{i 0}=m_{i 3}+m_{i 4}$. 
The time derivative is approximated by

$$
\frac{\partial f\left(x_{0}, y_{0}, n \Delta t\right)}{\partial t}=\frac{f_{0}^{n+1}-f_{0}^{n}}{\Delta t}+\mathcal{O}(\Delta t)
$$

Finally, the nonlocal term can be expressed by means of the Taylor series expansion as

$$
\begin{gathered}
\int_{\Omega} F\left(x+x_{0}, y+y_{0}\right) d \mathbf{x}=\int_{\Omega}\left(F\left(\mathbf{x}_{0}\right)+x \frac{\partial F}{\partial x}\left(\mathbf{x}_{0}\right)+y \frac{\partial F}{\partial y}\left(\mathbf{x}_{0}\right)\right. \\
\left.+\frac{x^{2}}{2} \frac{\partial^{2} F}{\partial x^{2}}\left(\mathbf{x}_{0}\right)+x y \frac{\partial^{2} F}{\partial x \partial y}\left(\mathbf{x}_{0}\right)+\frac{y^{2}}{2} \frac{\partial^{2} F}{\partial y^{2}}\left(\mathbf{x}_{0}\right)+R_{2}\right) d \mathbf{x}
\end{gathered}
$$

where $R_{2}$ is such that

$$
\lim _{(x, y) \rightarrow(0,0)} \frac{R_{2}}{\|(x, y)\|^{2}}=0 .
$$

In order to prove the main result of the paper concerning the conditional convergence of the GFD scheme for solving system (1) we need the following basic results.

Lemma 2.1. Let $M \in \mathfrak{M}_{n \times n}(\mathbb{R})$. If there exists some matrix norm such that $\|\boldsymbol{M}\|<1$, then

$$
\lim _{k \rightarrow \infty} \boldsymbol{M}^{k}=\boldsymbol{O}
$$

Lemma 2.2. Assume $\boldsymbol{M} \in \mathfrak{M}_{n \times n}(\mathbb{R})$, then the following statements are equivalent
i. $\lim _{k \rightarrow \infty} \boldsymbol{M}^{k}=\boldsymbol{O}$,
ii. $\rho(\boldsymbol{M})<1$,

where $\rho(\cdot)$ stands for the spectral radius. 


\section{GFDM explicit scheme}

Using the approximations given by (6), (7) and (8), we obtain the following 2-dimensional GFD explicit scheme:

$$
\left\{\begin{aligned}
u_{0}^{n+1}= & u_{0}^{n}+\Delta t\left[-m_{00} u_{0}^{n}+\sum_{i=1}^{s} m_{i 0} u_{i}^{n}-\chi\left(u_{0}^{n}\right)^{m}\left(-m_{00} v_{0}^{n}+\sum_{i=1}^{s} m_{i 0} v_{i}^{n}\right)\right] \\
& -\chi\left(u_{0}^{n}\right)^{m-1} \Delta t\left(-m_{01} u_{0}^{n}+\sum_{i=1}^{s}+m_{i 1} u_{i}^{n}\right)\left(-m_{01} v_{0}^{n}+\sum_{i=1}^{s} m_{i 1} v_{i}^{n}\right) \\
& -\chi\left(u_{0}^{n}\right)^{m-1} \Delta t\left(-m_{02} u_{0}^{n}+\sum_{i=1}^{s}+m_{i 2} u_{i}^{n}\right)\left(-m_{02} v_{0}^{n}+\sum_{i=1}^{s} m_{i 2} v_{i}^{n}\right) \\
& +\Delta t u_{0}^{n}\left[a_{0}-a_{1}\left(u_{0}^{n}\right)^{\alpha}+a_{2}\left(\left(u_{0}^{n}\right)^{\alpha}+\frac{\alpha}{2}\left(u_{0}^{n}\right)^{\alpha-1}\left(-\left(m_{01}+m_{02}\right) u_{0}^{n}\right.\right.\right. \\
& \left.+\sum_{i=1}^{s}\left(m_{i 1}+m_{i 2}\right) u_{i}^{n}\right)+\frac{\alpha(\alpha-1)}{6}\left(u_{0}^{n}\right)^{\alpha-2}\left(-m_{00} u_{0}^{n}+\sum_{i=1}^{s} m_{i 0} u_{i}^{n}\right) \\
& \left.\left.+\frac{\alpha(\alpha-1)}{4}\left(u_{0}^{n}\right)^{\alpha-2}\left(-m_{05} u_{0}^{n}+\sum_{i=1}^{s} m_{i 5} u_{i}^{n}\right)\right)\right]+\mathcal{O}\left(h_{i}^{2}, k_{i}^{2}\right) \\
v_{0}^{n+1}= & -m_{00} v_{0}^{n}+\sum_{i=1}^{s} m_{i 0} v_{0}^{n}-v_{0}^{n}+\left(u_{0}^{n}\right)^{\gamma}+\mathcal{O}\left(\Delta t\left(h_{i}^{2}, k_{i}^{2}\right)\right) .
\end{aligned}\right.
$$

Remark 3.1. From now, unless otherwise stated, $d=1$. Note that equation (9) holds since $\Omega=[0,1] \times[0,1]$. In other case the coefficients resulting from (8) may vary although the following theorem remains true. For an arbitrary and irregular domain we shall make

$$
\begin{gathered}
\int_{\Omega} F\left(x+x_{0}, y+y_{0}\right) d \boldsymbol{x}=F\left(\boldsymbol{x}_{0}\right)+\frac{\partial F}{\partial x}\left(\boldsymbol{x}_{0}\right) \int_{\Omega} x d \boldsymbol{x}+\frac{\partial F}{\partial y}\left(\boldsymbol{x}_{0}\right) \int_{\Omega} y d \boldsymbol{x} \\
+\frac{\partial^{2} F}{\partial x^{2}}\left(\boldsymbol{x}_{0}\right) \int_{\Omega} \frac{x^{2}}{2} d \boldsymbol{x}+\frac{\partial^{2} F}{\partial x \partial y}\left(\boldsymbol{x}_{0}\right) \int_{\Omega} x y d \boldsymbol{x}+\frac{\partial^{2} F}{\partial y^{2}}\left(\boldsymbol{x}_{0}\right) \int_{\Omega} \frac{y^{2}}{2} d \boldsymbol{x}+R_{2},
\end{gathered}
$$

and use numerical integration and interpolation if necessary to obtain the value of the integrals appearing in (10). The inclusion of such values in the numerical scheme (9) is a straightforward computation. 
The following result proves that the explicit scheme given by (9) is conditionally convergent for the fully parabolic case.

Theorem 3.1. Let $(U, V)$ be the exact solution of (1). Let $a_{0}, a_{1}>0$, $a_{2} \in \mathbb{R}, d=1$ and $m, \alpha, \gamma \geq 1$. Then, the GFD explicit scheme (9) is convergent if

$$
\Delta t<\frac{2}{m_{00}+\sum_{i=1}^{s}\left|m_{i 0}\right|+|\Phi|+|\Psi|}
$$


where

$$
\begin{aligned}
& \Phi:=-\chi m(m-1)\left(\left(m_{01}\right)^{2}+\left(m_{02}\right)^{2}\right) \xi_{1}^{m-2} \\
& +\chi m(m-1) \xi_{2}^{m-2} m_{01} v_{0}^{n} \sum_{i=1}^{s} m_{i 1} u_{i}^{n}+\chi m^{2} \xi_{3}^{m-1} m_{01} \sum_{i=1}^{s} m_{i 1} v_{i}^{n} \\
& -\chi m(m-1) \xi_{4}^{m-2}\left(\sum_{i=1}^{s} m_{i 1} u_{i}^{n}\right)\left(\sum_{i=1}^{s} m_{i 1} v_{i}^{n}\right)+\chi m(m-1) \xi_{5}^{m-2} m_{05} v_{0}^{n} \sum_{i=1}^{s} m_{i 2} u_{i}^{n} \\
& +\chi m^{2} \xi_{6}^{m-1} m_{02} \sum_{i=1}^{s} m_{i 2} v_{i}^{n}-\chi m(m-1) \xi_{7}^{m-2}\left(\sum_{i=1}^{s} m_{i 2} u_{i}^{n}\right)\left(\sum_{i=1}^{s} m_{i 2} v_{i}^{n}\right) \\
& \chi m \xi_{8}^{m-1} m_{00} v_{0}^{n}-\chi m \xi_{9}^{m-1} \sum_{i=1}^{s} m_{i 0} v_{i}^{n}+a_{0}-\left(a_{1}-a_{2}\right)(\alpha+1) \xi_{10}^{\alpha} \\
& -\frac{a_{2} \alpha(\alpha+1) \xi_{11}^{\alpha}}{2}\left(m_{01}+m_{02}\right)+\frac{a_{2} \alpha^{2} \xi_{12}^{\alpha-1}}{2} \sum_{i=1}^{s}\left(m_{i 1}+m_{i 2}\right) u_{i}^{n} \\
& -\frac{a_{2} \alpha^{2}(\alpha-1) \xi_{13}^{\alpha-1}}{6} m_{00}+\frac{a_{2} \alpha(\alpha-1)^{2} \xi_{14}^{\alpha-2}}{6} \sum_{i=1}^{s} m_{i 0} u_{i}^{n} \\
& -\frac{a_{2} \alpha^{2}(\alpha-1) \xi_{15}^{\alpha-2}}{4} m_{05}+\frac{a_{2} \alpha(\alpha-1)^{2} \xi_{16}^{\alpha-1}}{4} \sum_{i=1}^{s} m_{i 5} u_{i}^{n} \\
& +\left|\chi m\left(U_{0}^{n}\right)^{m-1} v_{0}^{n} m_{01} \sum_{i=1}^{s} m_{i 1}\right|+\left|\chi m\left(U_{0}^{n}\right)^{m-1} \sum_{i=1}^{s} m_{i 1} v_{i}^{n}\right| \sum_{i=1}^{s}\left|m_{i 1}\right| \\
& +\left|\chi m\left(U_{0}^{n}\right)^{m-1} v_{0}^{n} m_{02} \sum_{i=1}^{s} m_{i 2}\right|+\left|\chi m\left(U_{0}^{n}\right)^{m-1} \sum_{i=1}^{s} m_{i 2} v_{i}^{n}\right| \sum_{i=1}^{s}\left|m_{i 2}\right| \\
& \frac{\left|a_{2}\right| \alpha}{2}\left(U_{0}^{n}\right)^{\alpha} \sum_{i=1}^{s}\left|m_{i 1}+m_{i 2}\right|+\left|\frac{a_{2} \alpha(\alpha-1)\left(U_{0}^{n}\right)^{\alpha-1}}{6}\right| \sum_{i=1}^{s}\left|m_{i 0}\right| \\
& +\left|\frac{a_{2} \alpha(\alpha-1)\left(U_{0}^{n}\right)^{\alpha-1}}{4}\right| \sum_{i=1}^{s}\left|m_{i 5}\right|
\end{aligned}
$$


and

$$
\begin{aligned}
|\Psi| & :=\mid-\chi m\left(U_{0}^{n}\right)^{m}\left[\left(m_{01}\right)^{2}+\left(m_{02}\right)^{2}\right]+\chi m\left(U_{0}^{n}\right)^{m-1} m_{01} \sum_{i=1}^{s} m_{i 1} U_{i}^{n} \\
& +\chi m\left(U_{0}^{n}\right)^{m-1} m_{02} \sum_{i=1}^{s} m_{i 2} U_{i}^{n}-\chi\left(U_{0}^{n}\right)^{m} m_{00} \mid \\
& +\left|\chi m\left(U_{0}^{n}\right)^{m} m_{01}\right| \sum_{i=1}^{s}\left|m_{i 1}\right|+\left|\chi m\left(U_{0}^{n}\right)^{m-1} \sum_{i=1}^{s} m_{i 1} U_{i}^{n}\right| \sum_{i=1}^{s}\left|m_{i 1}\right| \\
& +\left|\chi m\left(U_{0}^{n}\right)^{m} m_{02}\right| \sum_{i=1}^{s}\left|m_{i 2}\right|+\left|\chi m\left(U_{0}^{n}\right)^{m-1} \sum_{i=1}^{s} m_{i 2} U_{i}^{n}\right| \sum_{i=1}^{s}\left|m_{i 2}\right| \\
& +\left|\chi\left(U_{0}^{n}\right)^{m}\right| \sum_{i=1}^{s}\left|m_{i 0}\right|,
\end{aligned}
$$

for some $\xi_{j} \in\left(u_{i}^{n}, U_{i}^{n}\right) \cap\left(U_{i}^{n}, u_{i}^{n}\right), \forall j \in\{1, \ldots, 16\}, \forall i \in\{0,1, \ldots, s\}$.

Proof. Consider the first equation of (9) (approximate solution) and subtract the same expression for the exact solution, i.e., in terms of $U_{i}^{n}$ and $V_{i}^{n}$ (which stands for the exact solution at time $n \Delta t$ ). Let us call $\tilde{u}_{i}^{n}:=u_{i}^{n}-U_{i}^{n}$ (similarly for $\tilde{v}_{i}^{n}$ ) and take the maximum of $\tilde{u}_{i}^{n}$ among all nodes of the star, that is, $\tilde{u}^{n}:=\max _{i=0, \ldots, s}\left|\tilde{u}_{i}^{n}\right|$. Then, after some straightforward computations (the reader may see [2] for further details of the computations) together with the Mean Value Theorem applied to the functions

$$
f(\lambda)=(\lambda)^{\delta}, \quad \delta=m, \alpha,
$$

we arrive to the following

$$
\tilde{u}^{n+1} \leq \tilde{u}^{n}\left[\left|1-\Delta t m_{00}\right|+\Delta t \sum_{i=1}\left|m_{i 0}\right|+\Delta t|\Phi|\right]+\Delta t|\Psi| \tilde{v}^{n}
$$

where $\Phi$ and $\Psi$ are defined in (12) and (13), respectively. If we perform the same computations with the second equation of (9) and its expression for the exact solution, it yields

$$
\frac{\tilde{v}_{0}^{n}-\tilde{v}_{0}^{n}}{\Delta t}=-\left(m_{00}+1\right) \tilde{v}_{0}^{n}+\sum_{i=1}^{s} m_{i 0} \tilde{v}_{i}^{n}+\gamma \xi_{17}^{\gamma-1} \tilde{u}_{0}^{n} .
$$


Again, by taking bounds and rewritting (15) in terms of $\tilde{u}^{n}, \tilde{v}^{n}$, we get

$$
\tilde{v}^{n+1} \leq \tilde{u}^{n} \Delta t \gamma \xi_{17}^{\gamma-1}+\tilde{v}^{n}\left[\left|1-\Delta t\left(m_{00}+1\right)\right|+\Delta t \sum_{i=1}^{s}\left|m_{i 0}\right|\right] .
$$

Therefore, inequalities (14) and (16) can be expressed as

$$
\left(\begin{array}{c}
\tilde{u}^{n+1} \\
\tilde{v}^{n+1}
\end{array}\right) \leq\left(\begin{array}{ll}
M_{11} & M_{12} \\
M_{21} & M_{22}
\end{array}\right)\left(\begin{array}{c}
\tilde{u}^{n} \\
\tilde{v}^{n}
\end{array}\right)
$$

for an obvious choice of $M_{l r}$, with $r, l \in\{1,2\}$. Convergence of the explicit scheme is assured if the eigenvalues of $\left(M_{r l}\right)$ are all smaller than 1 . Consider the $\|\cdot\|_{1}$ norm as the maximum sum by rows. Clearly, since $\Delta t<<1$, $\|M\|_{1}=M_{11}+M_{12}$ and, by assuming,

$$
\Delta t<\frac{2}{m_{00}+\sum_{i=1}^{s}\left|m_{i 0}\right|+|\Phi|+|\Psi|},
$$

we have that $\|M\|_{1}<1$. Therefore, Lemma 2.1 implies $\lim _{k \rightarrow \infty} M^{k}=\mathbf{0}$. Finally, by Lemma 2.2 we obtain that the spectral radius is less that 1 , which proves the result.

Remark 3.2. The above result covers only the parabolic-parabolic case with $d \neq 0$. The conditional convergence of the GFD explicit scheme for the parabolic-elliptic case, i.e., $d=0$, has been obtained in [2] by the authors.

\section{Numerical examples}

In this section we present numerical examples of the applicability of the GFD scheme given by (9) for solving the non-linear non-local system (1). For our simulations we use as time step $\Delta t=0.001$ and consider as discretization of the domain $\Omega=[0,1] \times[0,1]$ both regular and irregular clouds of points of Figure 1, each one with 437 nodes. We have chosen the potential weighting function $w=\frac{1}{d i s t^{4}}$ and performed our simulations using an 8-node star in all cases.

Notice that we have introduced some ficticious or vitual nodes (black nodes in Figure 1) in order to deal with the homogeneous Neumann boundary conditions,

$$
\frac{\partial U}{\partial \nu}=\frac{\partial V}{\partial \nu}=0
$$



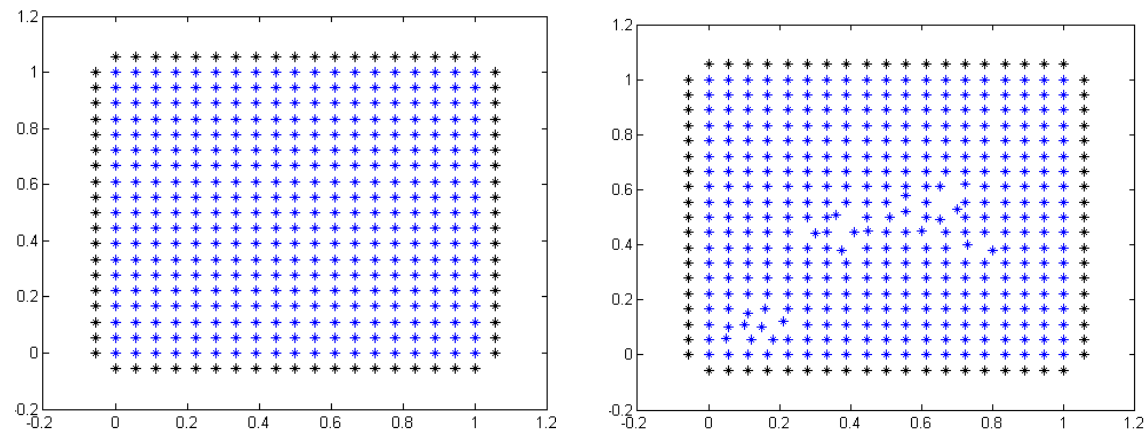

Figure 1: Regular and irregular clouds of points

since we use the central difference, which is of second order, if the domain $\Omega$ has regular boundary (in the sense of the distribution of nodes) and GFD formulae in other case. For all examples we compute the difference between the numerical solution and the constant steady states using the $l^{\infty}$ norm, that is,

$$
\|v\|_{l^{\infty}(\Omega)}=\max _{i=0, \ldots, N}\left\{\left|v_{i}\right|\right\} .
$$

In order to illustrate the accuracy of this meshless method, let us first find the steady states of the problem.

\subsection{Steady states}

In order to study the asymptotic behavior of system (1) we look at the constant steady states $\left(u^{*}, v^{*}\right)$. We assume, without loss of generality, that $|\Omega|=1$.

Lemma 4.1. Assume $a_{0}, a_{1}, \alpha, \gamma>0, a_{1}>\left|a_{2}\right|$ then the constant steady state of (1) is

$$
\left(u^{*}, v^{*}\right)=\left(\sqrt[\alpha]{\frac{a_{0}}{a_{1}-a_{2}}},\left(\sqrt[\alpha]{\frac{a_{0}}{a_{1}-a_{2}}}\right)^{\gamma}\right)
$$

Proof. Steady states of system (1) are the solutions to the homogeneous one

$$
\left\{\begin{array}{l}
0=a_{0}-a_{1}\left(u^{*}\right)^{\alpha}+a_{2} \int_{\Omega}\left(u^{*}\right)^{\alpha} d \mathbf{x}, \\
0=-v^{*}+\left(u^{*}\right)^{\gamma}
\end{array}\right.
$$




\begin{tabular}{cccccc}
\hline $\mathrm{T}(\mathrm{s})$ & 0 & 2.5 & 5 & 7.5 & 10 \\
\hline$\left\|\frac{\sqrt{2}}{2}-u\right\|_{l^{\infty}(\Omega)}$ & 1.2929 & 0.0024 & 0.0001 & $0 \mathrm{e}-03$ & $0 \mathrm{e}-04$ \\
\hline$\|0.5-v\|_{l^{\infty}(\Omega)}$ & 0.5000 & 0.0436 & 0.0036 & $0.2929 \mathrm{e}-03$ & $0.2401 \mathrm{e}-4$ \\
\hline
\end{tabular}

Table 1: Values of $\left\|\frac{\sqrt{2}}{2}-u\right\|_{l^{\infty}(\Omega)}$ and $\|0.5-v\|_{l^{\infty}(\Omega)}$ in the Example 1.

Since $|\Omega|=1$, the first equation lead us to

$$
\left(u^{*}\right)^{\alpha}=\frac{a_{0}}{a_{1}-a_{2}},
$$

and by the second equation we arrive to the result for $v^{*}$.

Remark 4.1. The above result covers the following two particular cases:

1. Clearly, if we make $\alpha=m=\gamma=1$ and $d=0$, the result is the one obtained by Negreanu and Tello [6].

2. Also, by making $d=1, a_{0}=r, a_{1}=\mu$ and $a_{2}=0$, Ding et al [3] obtained the convergence of the solution to the constant steady state $\left(u^{*}, v^{*}\right)$.

Asymptotic stability of the steady state has been obtained in both papers.

Remark 4.2. If $a_{0}, a_{2} \leq 0$, for any $a_{1}>0$, the solution converges asymptotically to 0, again in accordance with [3].

\subsection{Example 1}

In this first example we solve the case which appears in [3], that is to say, the case of Remark 4.1. Therefore we fix $a_{2}=0$. Consider $U_{0}(x, y)=2 x^{2}$ and $V_{0}(x, y)=\exp \left(-10\left[(x-1.2)^{2}+(y-1)^{2}\right]\right)$. Assume the following relation of parameters: $d=1, m=1, \alpha=2, \gamma=2, \chi=0.5$ and $a_{0}=2, a_{1}=4$. Then, by theory, we expect to find convergence towards $\left(\frac{\sqrt{2}}{2}, \frac{1}{2}\right)$. We consider the regular cloud of points of Figure 1.

Table 1 shows the values of the $l^{\infty}$ norm of the difference of the solution and the asymptotic value at different times, and Figure 2 the discrete solution at $0,2.5$ and 10 seconds. 

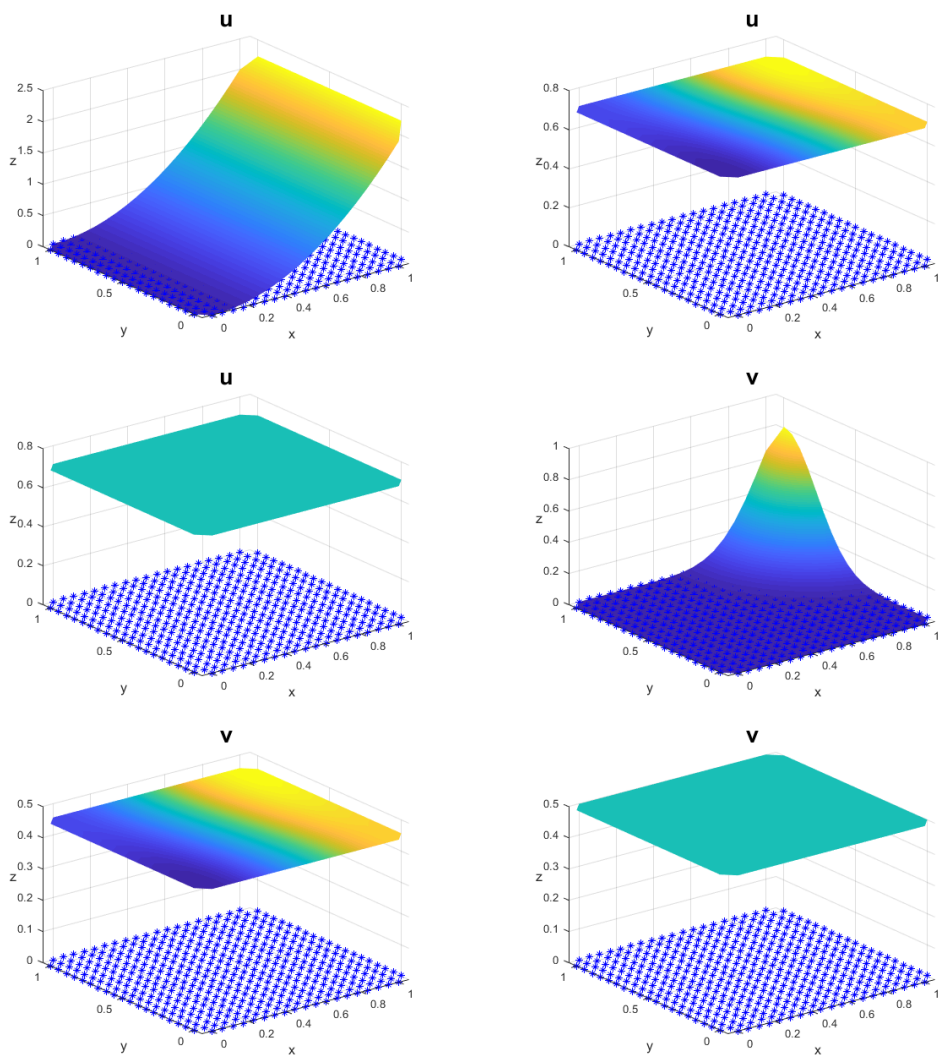

Figure 2: $u, v$-solution for 0, 2.5 and 10 seconds in the Example 1.

\subsection{Example 2}

We now consider $a_{0}=a_{2}=-1$ and $a_{1}=1$ for initial data $U_{0}(x, y)=$ $\exp \left(-10\left[(x-0.5)^{2}+(y-0.5)^{2}\right]\right)$ and $V_{0}(x, y)=0.5 \exp \left(-10\left[(x-0.2)^{2}+(y-\right.\right.$ $\left.1)^{2}\right]$ ) in the irregular cloud of points. Also we put $d=1, m=1, \alpha=2, \gamma=1$ and $\chi=0.5$. As stated in Remark 4.2 we expect to find asymptotic decay of the solution to 0 .

Table 2 presents the $l^{\infty}$ norm of the numerical solution at times $0,2.5$, 5, 7.5 and 10 seconds and Figure 3 the plot of the solution at $0,2.5$ and 10 seconds. As stated, solution tends to zero very rapidly.

\subsection{Example 3}

Consider as initial data $U_{0}(x, y)=\exp \left(-10\left[(x-0.1)^{2}+(y-0.1)^{2}\right]\right)$ and $V_{0}(x, y)=0.7 \exp \left(-10\left[(x-1.2)^{2}+(y-1)^{2}\right]\right)$. We choose the relation of 


\begin{tabular}{cccccc}
\hline $\mathrm{T}(\mathrm{s})$ & 0 & 2.5 & 5 & 7.5 & 10 \\
\hline$\|u\|_{l^{\infty}(\Omega)}$ & 0.9990 & 0.0234 & 0.0019 & 0.0002 & $0.0129 \mathrm{e}-03$ \\
\hline$\|v\|_{l^{\infty}(\Omega)}$ & 0.4975 & 0.0639 & 0.0100 & 0.0012 & $0.1321 \mathrm{e}-03$ \\
\hline
\end{tabular}

Table 2: Values of $\|u\|_{l^{\infty}(\Omega)}$ and $\|v\|_{l^{\infty}(\Omega)}$ in the Example 2.

\begin{tabular}{cccccc}
\hline $\mathrm{T}(\mathrm{s})$ & 0 & 2.5 & 5 & 7.5 & 10 \\
\hline$\|0.5-u\|_{l^{\infty}(\Omega)}$ & 1.4951 & 0.0366 & 0.0032 & 0.0003 & $0.0218 \mathrm{e}-3$ \\
\hline$\|0.5-v\|_{l^{\infty}(\Omega)}$ & 0.5000 & 0.1143 & 0.0172 & 0.0021 & $0.2247 \mathrm{e}-3$ \\
\hline
\end{tabular}

Table 3: Values of $\|0.5-u\|_{l^{\infty}(\Omega)}$ and $\|0.5-v\|_{l^{\infty}(\Omega)}$ in the Example 3.

parameters: $d=1, m=1, \alpha=1, \gamma=1$. As coefficients of the logistic term we take $a_{0}=1, a_{1}=3$ and $a_{2}=1$. Also, we take $\chi=0.5$. Hence, in accordance with (19) we expect to find convergence to $(1 / 2,1 / 2)$. Table 3 shows the maximum distance between the numerical solution and the limit value ( 0.5 for both $u$ and $v)$. Figure 4 shows the numerical solution at the initial time and 2.5 and 10 seconds.

\subsection{Example 4}

For this final example we choose the following initial data over the irregular cloud of points

$$
U_{0}(x, y)=e^{-10\left[(x-0.5)^{2}+(y-0.5)^{2}\right]}, \quad V_{0}(x, y)=1-0.5 e^{-10\left[(x-0.2)^{2}+(y-1)^{2}\right]} .
$$

Put $d=1, m=2, \alpha=2, \gamma=2, \chi=0.5$ and $a_{0}=3, a_{1}=8$ and $a_{2}=-1$. Then, $\left(u^{*}, v^{*}\right)=\left(\frac{\sqrt{3}}{3}, \frac{1}{3}\right)$.

Table 4 shows the differences, in $l^{\infty}$ norm, of the asymptotic limit and the discrete solution. In Figure 5 we plot the numerical solution at 0, 2.5 and 10 seconds. 

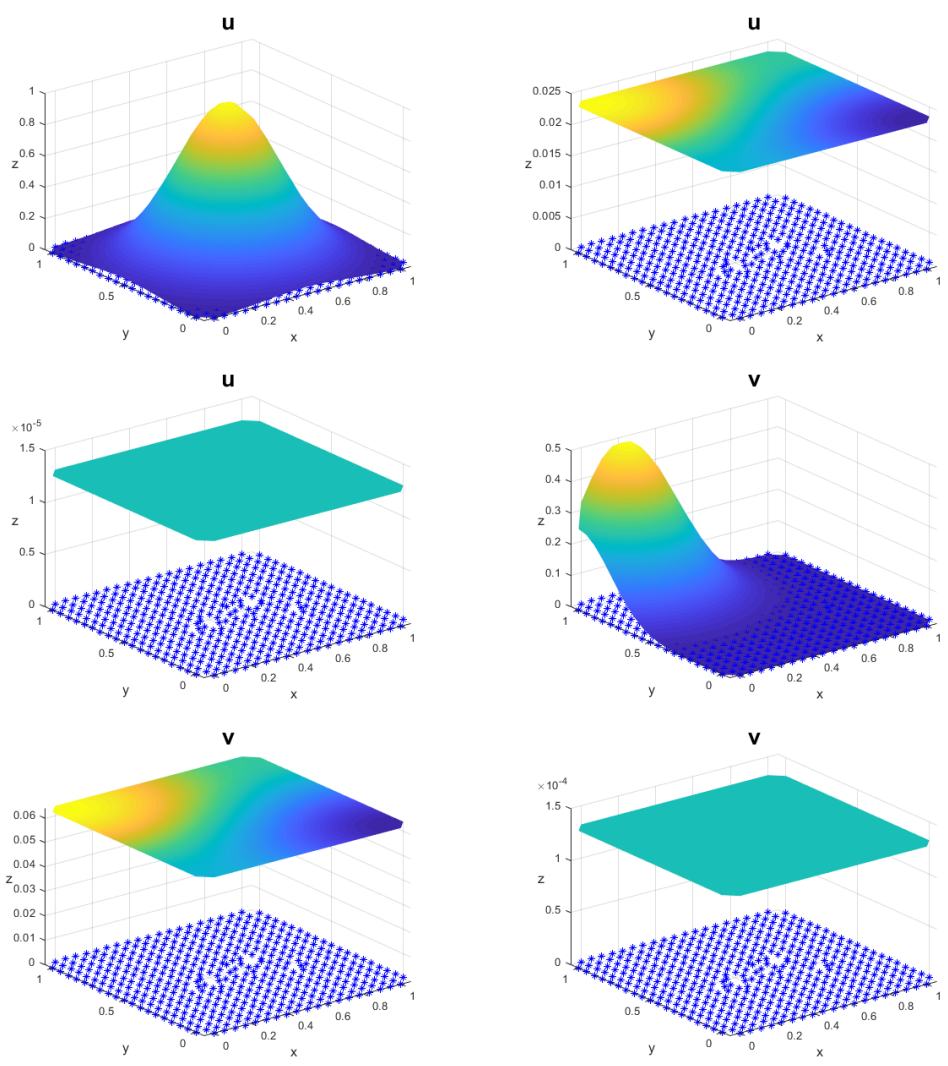

Figure 3: $u, v$-solution for $0,2.5$ and 10 seconds in the Example 2.

\begin{tabular}{cccccc}
\hline $\mathrm{T}(\mathrm{s})$ & 0 & 2.5 & 5 & 7.5 & 10 \\
\hline$\left\|\frac{\sqrt{3}}{3}-u\right\|_{l_{\infty}(\Omega)}$ & 0.5774 & 0.0003 & 0.0000 & $0 \mathrm{e}-03$ & $0 \mathrm{e}-04$ \\
\hline$\left\|\frac{1}{3}-v\right\|_{l^{\infty}(\Omega)}$ & 0.6667 & 0.0419 & 0.0034 & $0.2815 \mathrm{e}-03$ & $0.2308 \mathrm{e}-4$ \\
\hline
\end{tabular}

Table 4: Values of $\left\|\frac{\sqrt{3}}{3}-u\right\|_{l^{\infty}(\Omega)}$ and $\left\|\frac{1}{3}-v\right\|_{l^{\infty}(\Omega)}$ in the Example 4. 

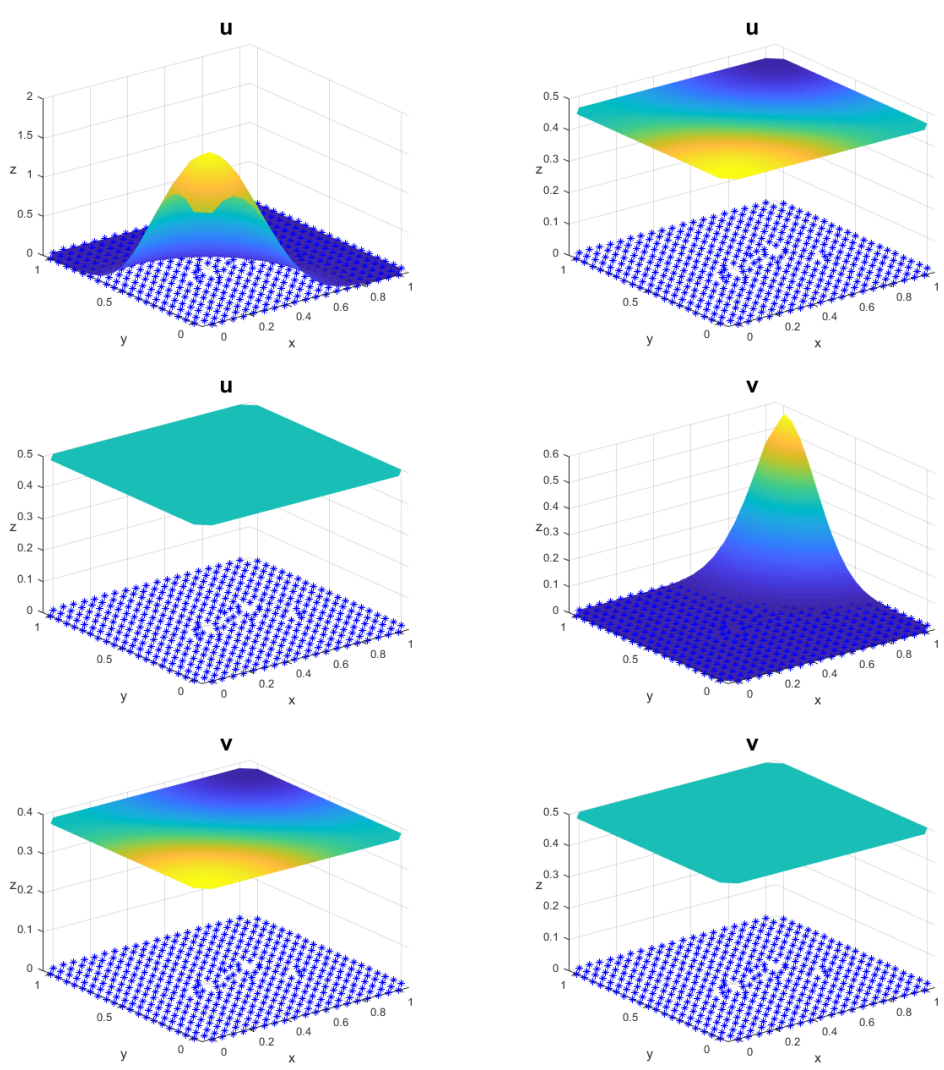

Figure 4: $u, v$-solution for 0, 2.5 and 10 seconds in the Example 3. 

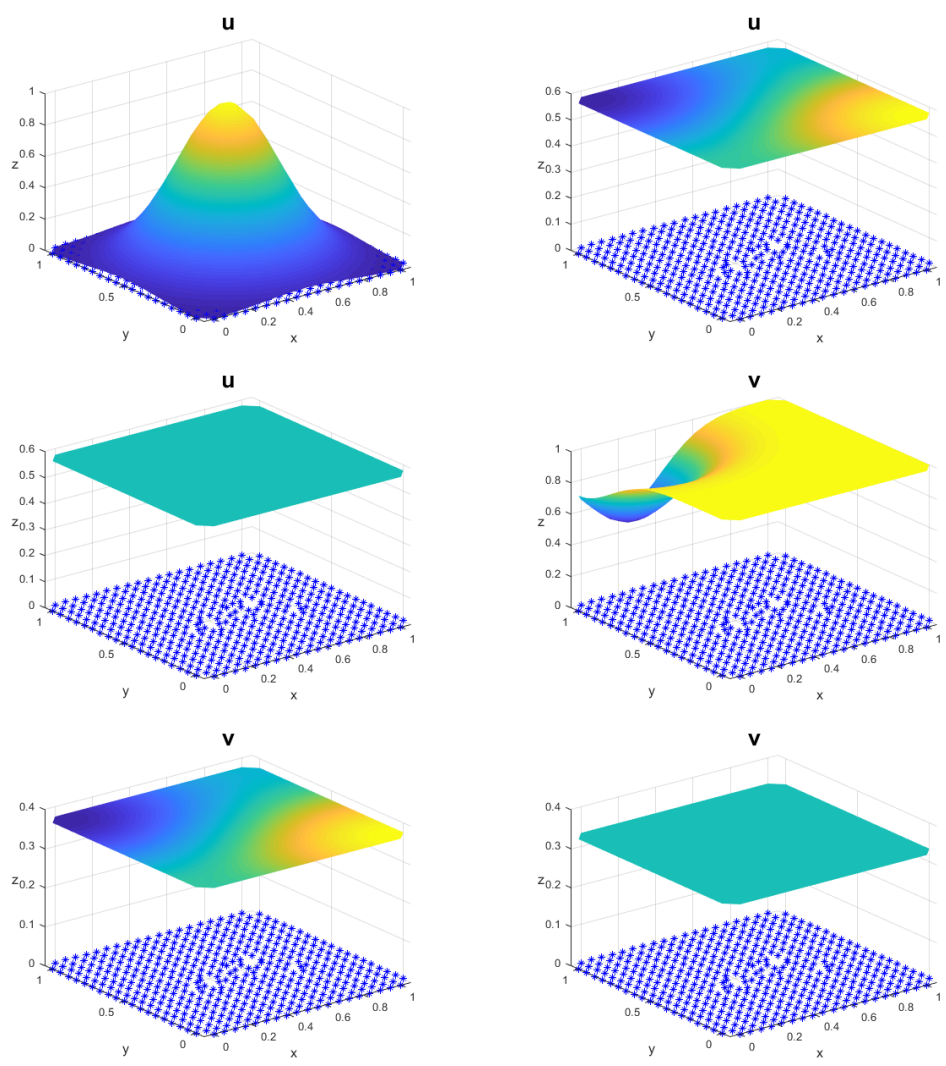

Figure 5: $u, v$-solution for 0, 2.5 and 10 seconds in the Example 4. 


\begin{tabular}{cccccc}
\hline $\mathrm{T}(\mathrm{s})$ & 0 & 2.5 & 5 & 7.5 & 10 \\
\hline$\left\|\frac{1}{4}-u\right\|_{l^{\infty}(\Omega)}$ & 0.75 & 0.0141 & 0.0011 & $8.9589 \mathrm{e}-05$ & $7.3423 \mathrm{e}-06$ \\
\hline$\left\|\frac{1}{4}-v\right\|_{l^{\infty}(\Omega)}$ & - & 0.0141 & 0.0011 & $8.9679 \mathrm{e}-05$ & $7.3497 \mathrm{e}-06$ \\
\hline
\end{tabular}

Table 5: Values of $\left\|\frac{1}{4}-u\right\|_{l^{\infty}(\Omega)}$ and $\left\|\frac{1}{4}-v\right\|_{l^{\infty}(\Omega)}$ in the Example 5.

\subsection{Example 5}

In this example we consider the parabolic-elliptic system (0.1) of [6] with $f=0, d=0$ and $\lambda=1$ (also $m=\alpha=\gamma=1$ ). We choose the irregular clouds of points of Fig.1 and initial data

$$
U_{0}(x, y)=1-e^{-10\left[(x-0.4)^{2}+(y-0.7)^{2}\right]}
$$

and parameters $a_{0}=a_{2}=1$ and $a_{1}=5$. Therefore, convergence towards $\left(\frac{1}{4}, \frac{1}{4}\right)$ is expected. This is clear from Table 5 , where we show the difference, computed in $l^{\infty}$ norm, between the constant steady state and the numerical solution. We plot in Figure 6 the approximate $u$ and $v$ solutions, so the asymptotic convergence of the continuous and the discrete model are patent. 

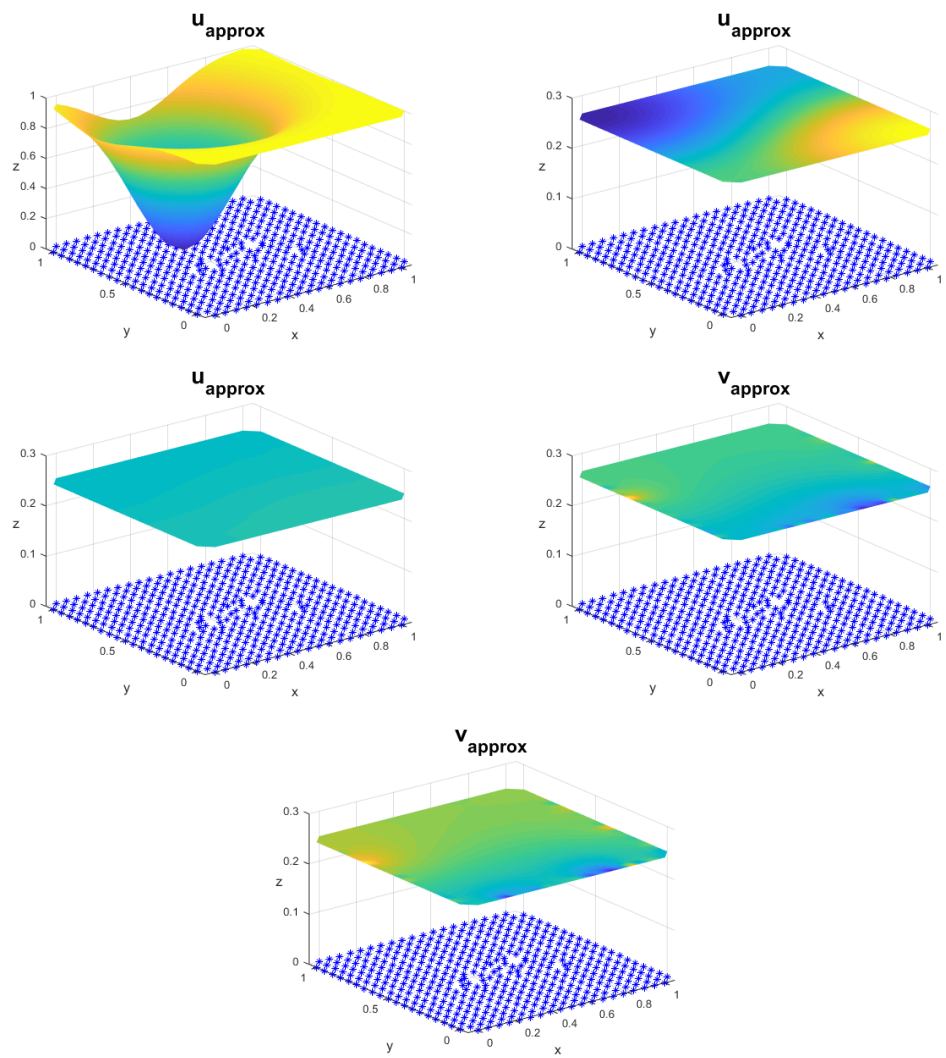

Figure 6: $u, v$-solution for 0, 2.5 and 10 seconds in the Example 5. 


\begin{tabular}{cccccc}
\hline $\mathrm{T}(\mathrm{s})$ & 0 & 2.5 & 5 & 7.5 & 10 \\
\hline$\|1-u\|_{l^{\infty}(\Omega)}$ & 1 & 0.0097 & $6.5381 \mathrm{e}-05$ & $4.3836 \mathrm{e}-07$ & $2.9387 \mathrm{e}-09$ \\
\hline$\|1-v\|_{l^{\infty}(\Omega)}$ & - & 0.0097 & $6.5512 \mathrm{e}-05$ & $4.3925 \mathrm{e}-07$ & $2.9487 \mathrm{e}-09$ \\
\hline
\end{tabular}

Table 6: Values of $\|1-u\|_{l^{\infty}(\Omega)}$ and $\|1-v\|_{l^{\infty}(\Omega)}$ in the Example 6 .

\subsection{Example 6}

In this example we also solve numerically the parabolic-elliptic system $(d=0)$, now with local competition among the individuals of the biological species. We put $a_{0}=2, a_{1}=1$ and $a_{2}=-1$, so the constant steady state in this case is $(1,1)$. We consider

$$
U_{0}(x, y)=e^{-10\left[(x-0.2)^{2}+(y-0.2)^{2}\right]}+e^{-10\left[(x-0.8)^{2}+(y-0.8)^{2}\right]}
$$

Table 6 shows the $l^{\infty}$ norm of the difference between the numerical solution and the steady state. Figure 7 shows the $u, v$-solutions at different times.

\subsection{Example 7}

Now we consider a more irregular domain, as seen in Figure 8. For this case, we take

$$
U_{0}(x, y)=2 e^{-10\left[(x-0.5)^{2}+(y-0.5)^{2}\right]}, \quad V_{0}(x, y)=3 e^{-10\left[(x-0.3)^{2}+(y-0.3)^{2}\right]},
$$

and parameters $a_{0}=3, a_{1}=7, a_{2}=4, d=\chi=m=\gamma=1$ and $\alpha=2$, so convergence towards $(1,1)$ is expected. We show in Table 7 the difference between these constant steady states and the numerical solution given by the GFDM. Also, we plot the solution for time $t=10 \mathrm{~s}$ in Figure 9.

\begin{tabular}{cccccc}
\hline $\mathrm{T}(\mathrm{s})$ & 0 & 2.5 & 5 & 7.5 & 10 \\
\hline$\|1-u\|_{\infty^{\infty}(\Omega)}$ & 1 & 0.0821 & 0.0073 & $5.3912 \mathrm{e}-04$ & $2.2126 \mathrm{e}-06$ \\
\hline$\|1-v\|_{l^{\infty}(\Omega)}$ & 1 & 0.0017 & $1.0181 \mathrm{e}-04$ & $1.3242 \mathrm{e}-05$ & $5.4523 \mathrm{e}-07$ \\
\hline
\end{tabular}

Table 7: Values of $\|1-u\|_{l^{\infty}(\Omega)}$ and $\|1-v\|_{l^{\infty}(\Omega)}$ in the Example 7. 

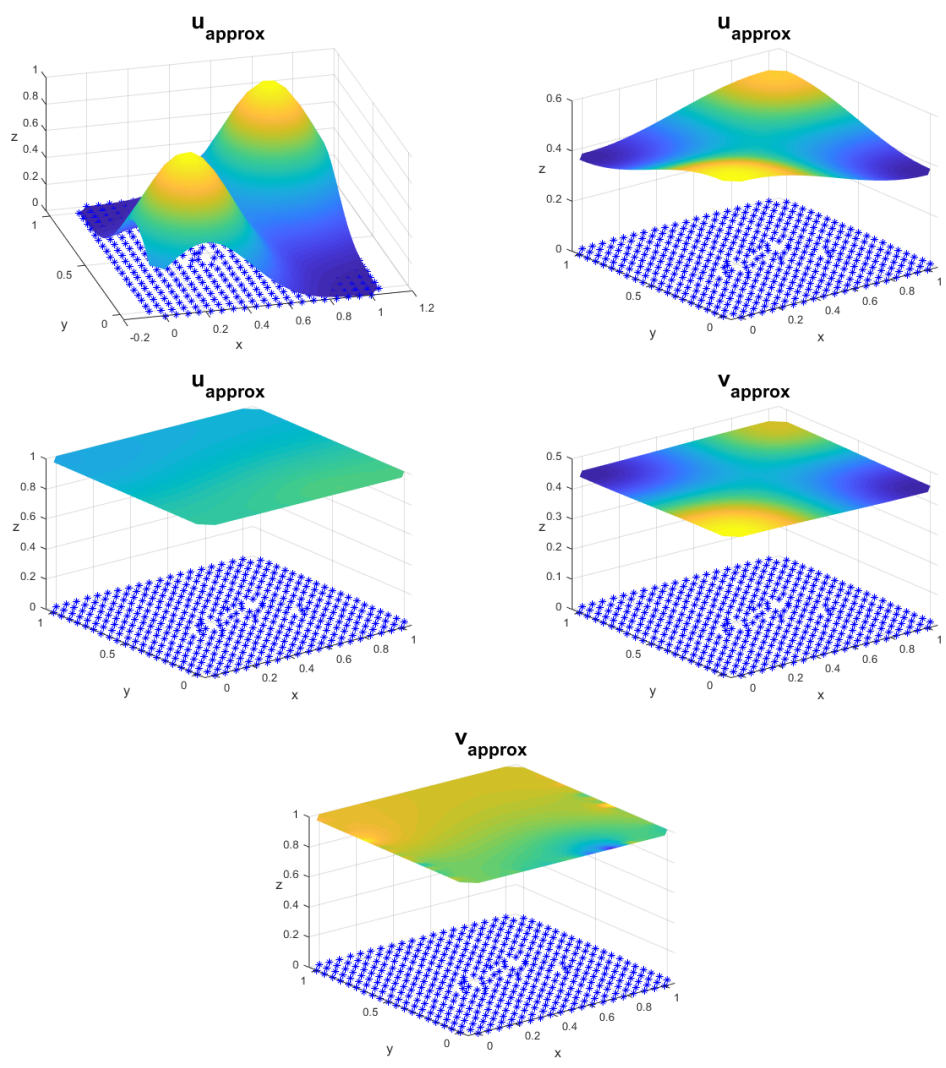

Figure 7: $u, v$-solution for $0,0.1$ and 2.5 seconds in the Example 6 .

\subsection{Example 8: influence of the time increment}

We present finally an example where we use different time increments, $\Delta t$, in order to show the effectiveness of the condition (11). We use the regular cloud of Figure 1, and for this distribution of nodes, for $\Delta t=0.02$ the scheme does not converge. We present the results when $\Delta t=0.0015$ and 0.0005 for times $t=1.5,3$ and 6 in Tables 8 and 9. For this computations we choose

$$
U_{0}(x, y)=2 e^{-10\left[(x-0.5)^{2}+(y-0.5)^{2}\right]}, \quad V_{0}(x, y)=3 e^{-10\left[(x-0.3)^{2}+(y-0.3)^{2}\right]},
$$




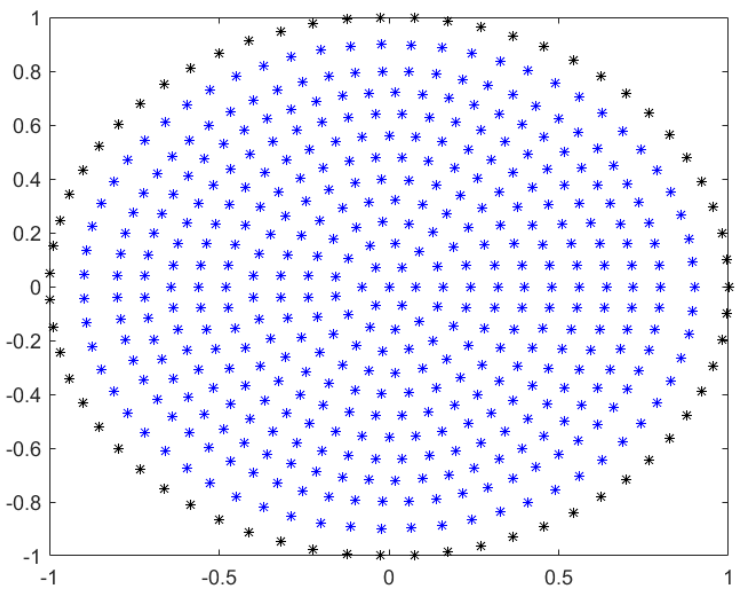

Figure 8: Irregular cloud of points used in the Example 7.

\begin{tabular}{cccc}
\hline $\mathrm{T}(\mathrm{s})$ & 1.5 & 3 & 6 \\
\hline$\left\|\sqrt{\frac{1}{3}}-u\right\|_{l^{\infty}(\Omega)}$ & 0.0913 & 0.0058 & $1.4448 \mathrm{e}-05$ \\
\hline$\left\|\sqrt{\frac{1}{3}}-v\right\|_{l^{\infty}(\Omega)}$ & 0.1806 & 0.0311 & $2.3221 \mathrm{e}-04$ \\
\hline
\end{tabular}

Table 8: Values of $\left\|\sqrt{\frac{1}{3}}-u\right\|_{l^{\infty}(\Omega)}$ and $\left\|\sqrt{\frac{1}{3}}-v\right\|_{l^{\infty}(\Omega)}$ for $\Delta t=0.0015$ in the Example 8.

and parameters $a_{0}=a_{2}=1, a_{1}=4, d=\chi=m=\gamma=1$ and $\alpha=2$. Therefore, the solutions tends to the steady state

$$
\left(\sqrt{\frac{1}{3}}, \sqrt{\frac{1}{3}}\right)
$$

As it is clear from the numerical evidence, to take a smaller time increment is translated in a more accurate. This fact validates formula (11). 

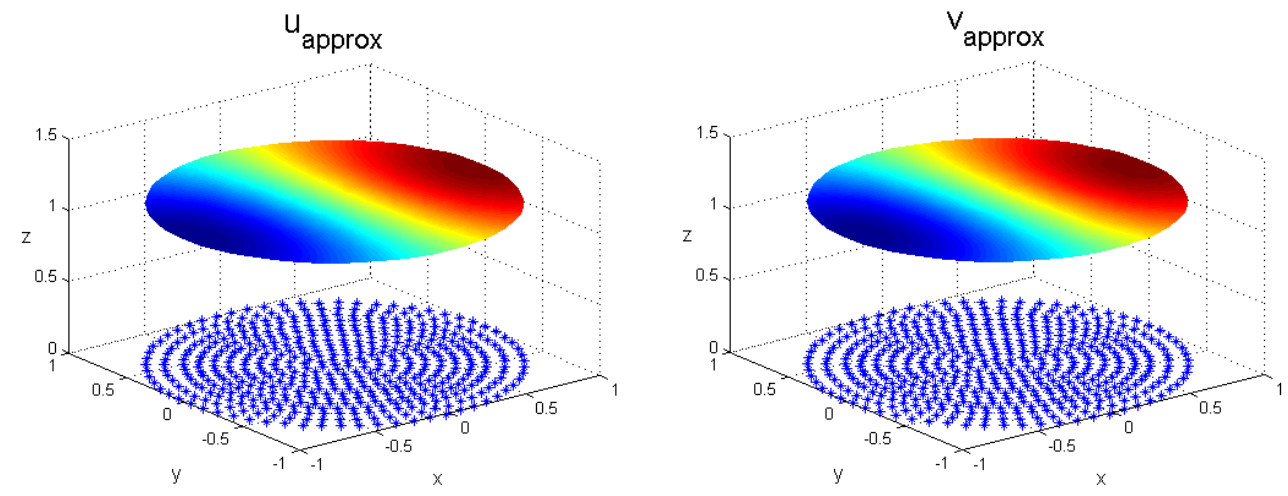

Figure 9: $u, v$-solution for $10 \mathrm{~s}$ in the Example 7.

\begin{tabular}{cccc}
\hline $\mathrm{T}(\mathrm{s})$ & 1.5 & 3 & 6 \\
\hline$\left\|\sqrt{\frac{1}{3}}-u\right\|_{l_{\infty}(\Omega)}$ & 0.0052 & $2.6433 \mathrm{e}-04$ & $6.5368 \mathrm{e}-07$ \\
\hline$\left\|\sqrt{\frac{1}{3}}-v\right\|_{l^{\infty}(\Omega)}$ & 0.2388 & 0.0015 & $7.439 \mathrm{e}-05$ \\
\hline
\end{tabular}

Table 9: Values of $\left\|\sqrt{\frac{1}{3}}-u\right\|_{l^{\infty}(\Omega)}$ and $\left\|\sqrt{\frac{1}{3}}-v\right\|_{l^{\infty}(\Omega)}$ for $\Delta t=0.0005$ in the Example 8. 


\section{Conclusions}

We have derived in Section 3 the discretization of the non-linear and nonlocal quasilinear system of parabolic PDEs in 2D using the GFDM. Also we have proved under which conditions the convergence can be expected. This meshless method does not reliance on the geometry of the domain or node distribution. Therefore it can be easily applied for solving highly non-linear PDEs over complicated and realistic domains.

All numerical results are in accordance with the theoretical asymptotic stability results. We have provided different simulations considering the most significant cases: first, the case no non-local interactions occur, and we have compared our numerical results with the asymptotic behavior obtained by Ding et al in [3]. Second, the case in which all coefficients of the logistic source are negative, obtaining that numerical solution decays to zero, also as stated in [3]. Finally we have extended our numerical study to the case in which individuals cooperate $\left(a_{2}>0\right)$ and compete $\left(a_{2}<0\right)$ in both parabolicparabolic and parabolic-elliptic cases, where the numerical solution indicates that a similar result as in [3] for non-local terms should be achieved and also the extension of the parabolic-elliptic to the fully parabolic problem considered by Negreanu and Tello in [6].

\section{Acknowledgements}

The authors acknowledge the support of the Escuela Técnica Superior de Ingenieros Industriales (UNED) of Spain, project 2019-IFC02, and of the Universidad Politécnica de Madrid (UPM) (Research groups 2019). This work is also partially support by the Project MTM2017-42907-P from MICINN (Spain).

\section{References}

[1] Anderson A.R., Chaplain M.A., Continuous and discrete mathematical models of tumor-induced angiogenesis. Bull Math Biol., 60 (5), 857-899, (1998).

[2] Benito J. J., Garcia A., Gavete L., Negreanu M., Ureña F., Vargas A. M., On the numerical solution to a parabolic-elliptic system with chemotactic 
and periodic terms using Generalized Finite Differences. Engineering Analysis with Boundary Elements, 113C, 181-190 (2020).

[3] Ding M., Wang W., Zhou S., Zheng S., Asymptotic stability in a fully parabolic quasilinear chemotaxis model with general logistic source and signal production. Journal of Differential Equations (2019), https://doi.org/10.1016/j.jde.2019.11.052.

[4] Horstmann D., From 1970 until present: the Keller-Segel model in chemotaxis and its consequences, Jahresbericht der Deutschen Mathematiker-Vereinigung. 105 (3), (2003), 103-165.

[5] Keller EF., Segel LA. Initiation of slime mold aggregation viewed as an instability. J. Theoret. Biol. 26, 399-415, (1970).

[6] Negreanu M., Tello J.I., On a competitive system under chemotactic effects with non-local terms. Nonlinearity, 26 (4), 1083-1103 (2013).

[7] Negreanu M., Tello J.I., Vargas A.M. (2017), On a Parabolic-Elliptic chemotaxis system with periodic asymptotic behavior, Mathematical Methods in Applied Sciences, https://doi.org/10.1002/mma.5423.

[8] Negreanu M., Tello J.I., Vargas A.M. (2020), On a fully Parabolic chemotaxis system with source term and periodic asymptotic behavior, to appear in Zeitschrift für angewandte Mathematik und Physik.

[9] Szymanska Z., Morales Rodrigo C., Lachowicz M., Chaplain M.A.J., Mathematical modelling of cancer invasion of tissue the role and effect of nonlocal interactions. Mathematical Models and Methods in Applied Sciences, 19 (2), 257-281 (2009).

[10] Ureña F., Gavete L., Benito J.J., García A., Vargas A.M., Solving the telegraph equation in 2-D and 3-D using generalized finite difference method (GFDM). Engineering Analysis with Boundary Elements, 112, 13-24, (2020).

[11] Ureña F., Gavete L., Garcia A., Benito J. J., Vargas A. M., Solving second order non-linear parabolic PDEs using generalized finite difference method (GFDM), Journal of Computational and Applied Mathematics, 354, (2019), 221-241. 